\title{
Efeito Analgésico Residual do Fentanil em Pacientes Submetidos a Revascularização do Miocárdio com Circulação Extracorpórea
}

\section{Residual Analgesic Effect of Fentanyl in Patients Undergoing Myocardial Revascularization with Cardiopulmonary Bypass}

Adriana Machado Issy ${ }^{1}$, Eloísa Bonetti Espada, TSA ${ }^{2}$, Rioko Kimiko Sakata, TSA ${ }^{1}$, Vera Lúcia Lanchote ${ }^{3}$, José Otávio Costa Auler Júnior, TSA ${ }^{4}$, Silvia Regina C. J. Santos ${ }^{5}$

\section{RESUMO}

Issy AM, Espada EB, Sakata RK, Lanchote VL, Auler Jr JOC, Santos SRCJ - Efeito Analgésico Residual do Fentanil em Pacientes Submetidos a Revascularização do Miocárdio com Circulação Extracorpórea

\begin{abstract}
Justificativa e Objetivos - Apesar do uso freqüente do fentanil em altas doses para operações de grande porte, a contribuição da concentração plasmática residual desse fármaco sobre a analgesia pós-operatória merece maiores investigações. O objetivo deste estudo é avaliar o efeito analgésico do fentanil residual no primeiro e segundo dias após revascularização miocárdica, bem como quantificar sua concentração.

Método - Foram investigados 11 pacientes submetidos a revascularização do miocárdio com circulação extracorpórea, sob anestesia geral empregando-se $50 \mu \mathrm{g}_{\mathrm{kg}}{ }^{-1}$ de fentanil, por via venosa. Avaliou-se a analgesia pela escala numérica verbal nos tempos zero (extubação), 70 minutos, 3, 5, 8 e 12 horas no primeiro dia e nos tempos zero (24 horas após extubação), 70 minutos, 3, 5, 8 e 12 horas no segundo dia. A dor foi avaliada mediante tosse vigorosa e fisioterapia respiratória. A cada mensuração da dor, questionou-se sobre a necessidade de o paciente receber complementação analgésica. As amostras plasmáticas do fentanil foram coletadas nos tempos zero, 70 minutos, 3, 5, 8 e 12 horas do primeiro e segundo dia de pós-operatório e mensuradas pelo método de radioimunoensaio.
\end{abstract}

Resultados - Aintensidade da dor variou em média de 1,9 a 3,7 no primeiro dia e de 2,1 a 3,8 no segundo dia de pós-operatório Os níveis plasmáticos de fentanil (>1 ng/ml), evidenciaram sua contribuição na analgesia no primeiro dia após a operação.

Conclusões - Apesar de não ter sido observada correlação entre a concentração plasmática residual de fentanil e a intensidade da dor, os pacientes apresentaram dor leve durante todo o período investigado.

\footnotetext{
* Recebido do (Received from) Instituto do Coração da Universidade de São Paulo, São Paulo, SP

1. Professora Adjunta de Anestesiologia da UNIFESP - São Paulo - SP

2. Diretora do Serviço de Anestesiologia do Hospital Universitário da Universidade de São Paulo - USP - São Paulo - SP

3. Professor Associado de Toxicologia da Universidade de São Paulo USP - Ribeirão Preto - São Paulo

4. Professor Titular de Anestesiologia da Universidade de São Paulo USP - São Paulo -SP

5. Professora Titular de Farmacoterapia da Universidade de São Paulo USP - São Paulo -SP

Apresentado (Submitted) em 24 de outubro de 2001

Aceito (Accepted) para publicação em 25 de janeiro de 2002

Correspondência para (Mail to):

Dra. Adriana Machado Issy

Rua Nova York, 539/81 - Brooklin

04560-001 São Paulo, SP

(C) Sociedade Brasileira de Anestesiologia, 2002
}

UNITERMOS: ANALGÉSICOS, Opióides: fentanil; CIRURGIA, Cardíaca: revascularização do miocárdio; DOR, Aguda: pós-operatória; TÉCNICAS DE MEDIÇÃO: escala numérica verbal

\section{SUMMARY}

Issy AM, Espada EB, Sakata RK, Lanchote VL, Auler Jr JOC, Santos SRCJ - Residual Analgesic Effect of Fentanyl in Patients Undergoing Myocardial Revascularization with Cardiopulmonary Bypass

Background and Objectives - In spite of the frequent use of high doses of fentanyl for major surgeries, the correlation of its residual plasma concentration to postoperative analgesia deserves further investigation. This study aimed at evaluating the residual analgesic effect of fentanyl in the first and second postoperative days of myocardial revascularization, as well as quantifying its concentration.

Methods - Participated in this study 11 patients undergoing myocardial revascularization with cardiopulmonary bypass under general anesthesia with $50 \mu \mathrm{g} . \mathrm{kg}^{-1}$ intravenous fentanyl. Analgesia was evaluated by a numeric verbal scale at moments zero (extubation), $70 \mathrm{~min}, 3,5,8$ and 12 hours in the first day; and moments zero (24 h after extubation), $70 \mathrm{~min}, 3,5,8$ and 12 hours in second post-operative day. Pain intensity to vigorous cough and respiratory physical therapy was evaluated. At every measurement, patients were asked about the need for analgesic complementation. Plasma samples were collected in moments zero (extubation), $70 \mathrm{~min}, 3,5,8$ and 12 hours during the first and second postoperative days for fentanyl radioimmunoassay.

Results - Mean pain intensity varied from 1.9 to 3.7 in the first day and from 2.1 to 3.8 in the second postoperative day. Fentanyl plasma levels (> $1 \mathrm{ng} / \mathrm{ml}$ ) evidenced its contribution to post-operative analgesia during the first postoperative day.

Conclusions - In spite of the lack of correlation between residual fentanyl plasma concentration and pain intensity, patients referred only mild pain during the whole investigation period.

KEY WORDS - ANALGESICS, Opioids: fentanyl; MEASUREMENT TECHNIQUES: numeric verbal scale; PAIN, Acute: postoperative; SURGERY, Cardiac: myocardial revascularization

\section{INTRODUÇÃO}

$\mathrm{O}$ fentanil é um opióide potente, utilizado com freqüência para anestesia geral ${ }^{1,2}$. Como vantagens, há sua rápida ação com manutenção da estabilidade cardiovascular, além de não provocar liberação de histamina ${ }^{3,4}$.

Comercialmente encontrado como sal citrato, sob a forma de solução aquosa, apresenta alta lipossolubilidade com coeficiente de partição octanol/água de 816, fator determi- 
nante da velocidade de entrada e saída do fármaco nos órgãos e tecidos, especialmente no sistema nervoso central. Essa facilidade de penetração é 156 vezes maior do que a da morfina ${ }^{1,5-7}$

O emprego de fentanil em altas doses (50 a $100 \mu \mathrm{g} . \mathrm{kg}^{-1}$ ) para operações de grande porte merece maiores investigações quanto à contribuição da concentração plasmática residual desse fármaco sob a analgesia pós-operatória.

O objetivo deste estudo foi avaliar o efeito analgésico residual do fentanil no pós-operatório de revascularização miocárdica e quantificar a concentração plasmática do fármaco nesse mesmo período.

\section{MÉTODO}

Após aprovação pela Comissão de Ética e da assinatura do Termo de Consentimento pelos pacientes, foram investigados 11 pacientes adultos de ambos os sexos portadores de insuficiência coronariana com indicação de revascularização do miocárdio. Foram excluídos os pacientes com doenças renal, hepática, endócrina, pulmonar ou neuromuscular. Os pacientes deveriam apresentar função cardíaca compensada e radiografia de tórax pré-operatória dentro dos limites da normalidade.

Uma hora antes da operação, os pacientes receberam como medicação pré-anestésica midazolam (7,5 e $15 \mathrm{mg})$ por via oral.

A monitorização foi feita através de cardioscopia, oximetria, pressão arterial invasiva, pressão venosa central e sondagem vesical. A indução da anestesia foi feita com $50 \mu \mathrm{g} \cdot \mathrm{kg}^{-1}$ de fentanil, por via venosa, seguida de bloqueador neuromuscular (pancurônio - 0,08 mg. $\mathrm{kg}^{-1}$ ) e a manutenção foi com isoflurano/ $\mathrm{O}_{2}$.

Acirculação extracorpórea foi feita com diluição total com solução de Ringer e hipotermia moderada ou normotermia. Para reversão da anticoagulação foi administrado sulfato de protamina até normalização do tempo de coagulação ativado (TCA).

Durante o ato anestésico-cirúrgico, foram utilizados fármacos vasoativos, conforme o estado hemodinâmico de cada paciente.

O diclofenaco foi usado por via muscular a cada 12 horas, sendo a primeira dose administrada após a extubação , conforme rotina estabelecida pelo Serviço.

O tempo zero do primeiro dia de pós-operatório, para avaliação da analgesia e para dosagem plasmática de fentanil, foi considerado o momento da extubação. No segundo dia de pós-operatório, considerou-se como tempo zero, 24 horas após o tempo zero do primeiro dia. A dor foi mensurada durante tosse vigorosa e fisioterapia respiratória nos tempos zero, $70 \mathrm{~min}, 3,5$, 8 e $12 \mathrm{~h}$ do primeiro dia e zero (24 h após a extubação), 70 min, 3, 5, 8 e 12 h do segundo dia. A coleta de sangue foi feita nesses mesmos tempos.

A cada mensuração da dor, questionou-se o paciente da necessidade de receber complementação analgésica. Nos casos de resposta positiva, foi administrada dipirona na dose de $1000 \mathrm{mg}$ por via venosa. Se ainda assim persistisse a dor, a complementação era feita com $100 \mathrm{mg}$ de tramadol por via venosa, diluídos em $50 \mathrm{ml}$ de solução de glicose a $5 \%$. Aos pacientes que apresentaram náusea após o uso de tramadol, foi administrado metoclopramida por via venosa. Persistindo a dor foram usados $30 \mathrm{mg}$ de meperidina também por via venosa.

A dor foi avaliada pela Escala Numérica Verbal (ENV). A escala numérica verbal registra a intensidade da dor, através do questionamento no momento da avaliação, sendo zero (ausência de dor) e 10 (pior dor possível).

As concentrações plasmáticas residuais de fentanil foram determinadas pelo método de radioimunoensaio em um contador específico de radiação $\beta$. O limite de quantificação do método foi de $50 \mathrm{pg} \cdot \mathrm{ml}^{-1}$.

Os dados foram submetidos a estudo estatístico, utilizando-se o software GraphPad Instat e GMC. Realizou-se o teste de Qui-quadrado para o estudo da distribuição de freqüência e aplicou-se o teste não paramétrico de Wilcoxon para comparação das médias e medianas dos períodos pré e pós-operatórios nas variáveis que não apresentaram distribuição normal. O nível de significância foi fixado em $p<0,05^{8}$.

\section{RESULTADOS}

Foram avaliados 7 homens e 4 mulheres com média de idade de 52,6 $\pm 8,4$ anos.

As médias das concentrações plasmáticas residuais de fentanil variaram entre 1,7 e 1,1 ng. $\mathrm{ml}^{-1}$ no primeiro dia e 0,7 e $0,3 \mathrm{ng} \cdot \mathrm{ml}^{-1}$ no segundo dia de pós-operatório, apresentando significância estatística (teste de Wilcoxon, $p<0,05$ ) em todos os tempos investigados, conforme ilustra a tabela I.

Tabela I - Concentração Plasmática do Fentanil (ng. $\mathrm{ml}^{-1}$ ) no Período Pós-Operatório

\begin{tabular}{lcc}
\hline Tempo $(\mathrm{h})$ & \multicolumn{2}{c}{ Mediana Média $(\mathrm{IC9} \%)$} \\
\hline 0 & $1^{\circ} \mathrm{PO}$ & $2^{\circ} \mathrm{PO}$ \\
\hline \multirow{2}{*}{1,2} & 1,7 & $0,5^{*}$ \\
& $1,6(1,2-2,0)$ & $0,6(0,4-0,9)$ \\
3 & 1,6 & $0,7^{*}$ \\
& $1,5(1,0-1,9)$ & $0,6(0,4-0,8)$ \\
5 & $1,4(0,6-2,6)$ & $0,6^{*}$ \\
& 1,3 & $0,6(0,4-0,9)$ \\
8 & $1,2(0,9-1,5)$ & $0,5^{*}$ \\
& 1,1 & $0,4\left(0,3^{*}-0,7\right)$ \\
12 & $1,2(0,8-1,5)$ & $0,3^{*}$ \\
\hline
\end{tabular}

$1^{\circ} \mathrm{PO}$ : primeiro dia de pós-operatório, $\mathbf{2}^{\circ} \mathrm{PO}$ : segundo dia de pós-operatório, IC95\%: intervalo de confiança $95 \%$; * significância estatística, teste de Wilcoxon, $p<0,05$

Ador (mediana) mensurada pela escala numérica verbal variou de 1,9 a 3 no primeiro dia e de 3,7 a 2,1 no segundo dia de pós-operatório, sem significância estatística (teste de Wilcoxon, $\mathrm{p}<0,05$ ) conforme ilustra a figura 1 e tabela II. 


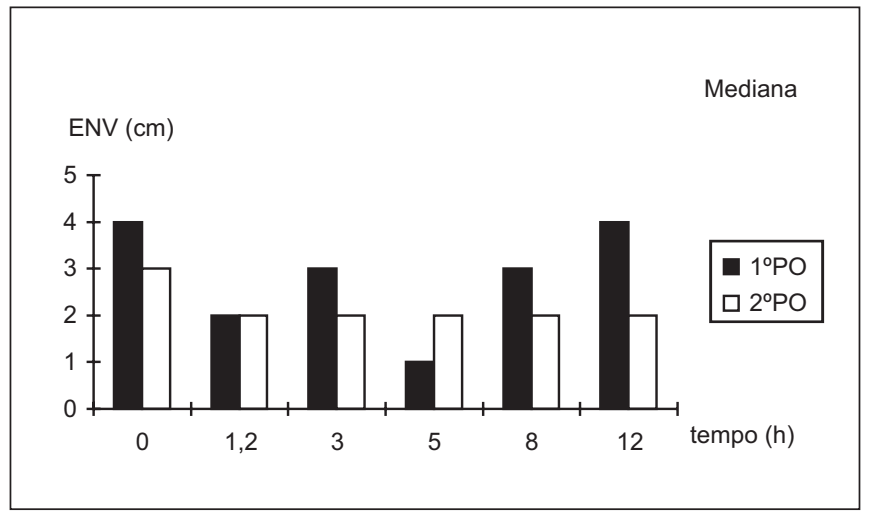

Figura 1 - Intensidade da Dor no Período Pós-Operatório $\left(1^{\circ}\right.$ e $2^{\circ}$ dias) dos Pacientes Submetidos à Cirurgia Cardíaca; sem significância estatística

Tabela II - Intensidade da Dor Medida pela Escala Numérica Verbal no $1^{\circ}$ e $2^{\circ}$ Dias de Pós-Operatório

\begin{tabular}{|c|c|c|}
\hline \multirow[t]{2}{*}{ Tempo (h) } & \multicolumn{2}{|c|}{ Mediana - Média (IC95\%) } \\
\hline & $1^{\circ} \mathrm{PO}$ & $2^{\circ} \mathrm{PO}$ \\
\hline 0 & $\begin{array}{c}4,0 \\
3,7(2,3-5,0)\end{array}$ & $\begin{array}{c}3,0 \\
3,8(2,3-5,2)\end{array}$ \\
\hline 1,2 & $\begin{array}{c}2,0 \\
2,8(2,0-3,5)\end{array}$ & $\begin{array}{c}2,0 \\
2,3(1,1-3,5)\end{array}$ \\
\hline 3 & $\begin{array}{c}3,0 \\
2,6(1,5-3,7)\end{array}$ & $\begin{array}{c}2,0 \\
2,1(0,95-3,3)\end{array}$ \\
\hline 5 & $\begin{array}{c}1,0 \\
1,9(0,7-3,0)\end{array}$ & $\begin{array}{c}2,0 \\
2,7(1,6-3,8)\end{array}$ \\
\hline 8 & $\begin{array}{c}3,0 \\
2,6(1,6-3,7)\end{array}$ & $\begin{array}{c}2,0 \\
2,2(0,9-3,5)\end{array}$ \\
\hline
\end{tabular}

IC95\%: intervalo de confiança 95\%, sem significância estatística, teste de Wilcoxon $p<0,05$

Correlacionou-se a intensidade da dor com a concentração plasmática residual de fentanil através da curva de Efeito versus Concentração no período pós-operatório, obtendo coeficientes de correlação de 0,04 para o primeiro dia e 0,0002 para o segundo dia de pós-operatório conforme a figura 2. A tabela III mostra o número de pacientes que receberam complementação analgésica, o tipo de medicação, o tempo em que foi administrado e a intensidade da dor durante o primeiro e segundo dias de pós-operatório.

\section{DISCUSSÃO}

O pré-requisito para investigação da concentração plasmática residual de fentanil é a disponibilidade de métodos analíticos com sensibilidade e especificidade para quantificar as baixas concentrações plasmáticas do fármaco.

Dos vários métodos citados na literatura, o método cromatografia gasosa com espectro de massa (CG-MS), apesar de apresentar sensibilidade, especificidade e linearidade adequadas, apresenta algumas desvantagens quando comparado ao método de radioimunoensaio (RIE), tais como: alto custo, laborioso procedimento de preparo da amostra e utilização em dobro do volume do material biológico no procedimento de extração ${ }^{4,9}$.

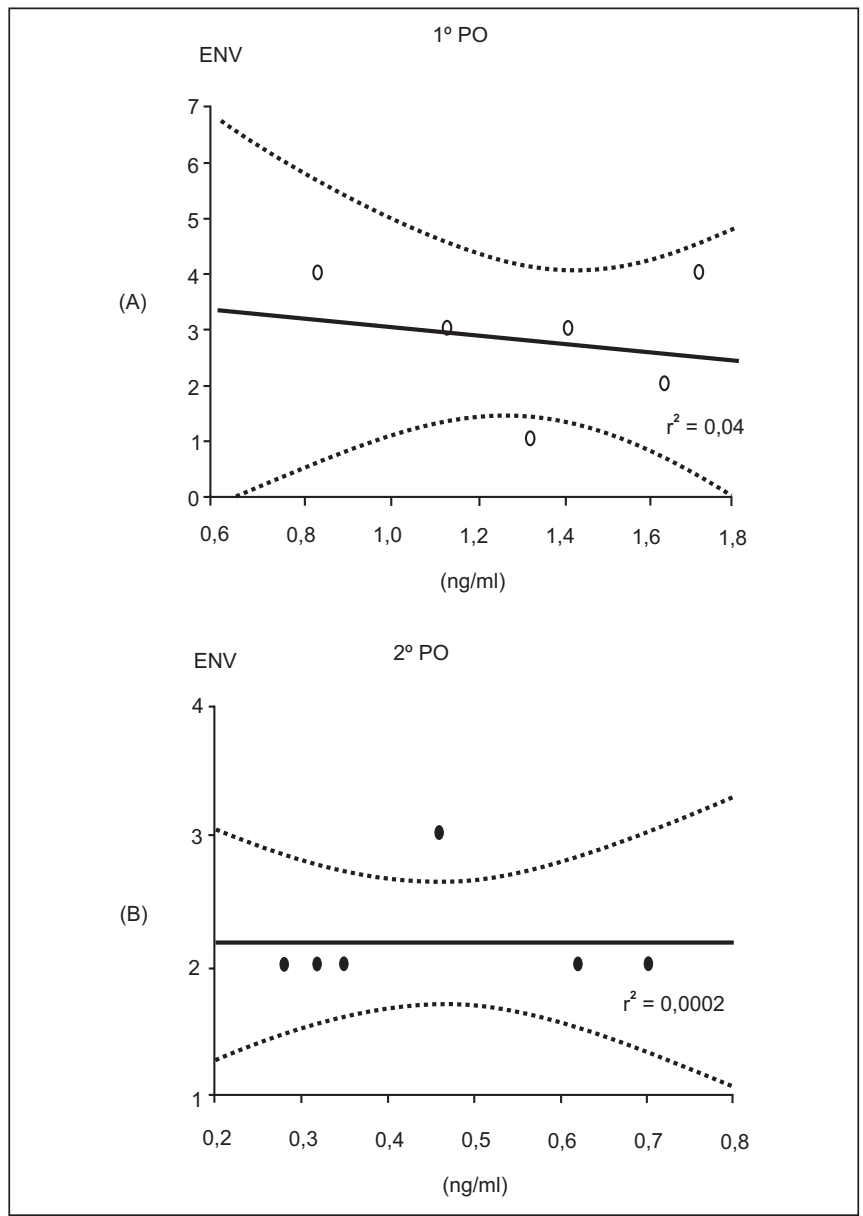

Figura 2 - Intensidade da Dor versus Concentração Plasmática Residual de Fentanil no Primeiro Dia (A) e no Segundo Dia (B) de Pós-Operatório

Atualmente, o método radioimunoensaio tem-se destacado não só pela alta sensibilidade, mas também pela fácil e rápida execução, com diminuição de erros analíticos, sendo, por isso, o método de escolha para a dosagem do opióide. Nessa técnica, o estabelecimento, a execução e a padronização dos procedimentos são essenciais para obtenção de resultados precisos e exatos ${ }^{4,10}$.

Na presente investigação, todos os pacientes foram submetidos a revascularização do miocárdio com circulação extracorpórea, procedimento este que, geralmente, envolve a colocação de 2 a 3 drenos torácicos ${ }^{11,12}$. De acordo com a experiência clínica, baseada na queixa dos pacientes, a presença desses drenos é responsável por dor intensa.

Embora seja subjetiva, a dor pode ser avaliada pelas escalas que permitem quantificar sua intensidade. Alguns autores 13,14 ressaltaram ainda os cuidados a serem tomados na escolha do método de avaliação da dor.

Estudo comparativo de seis métodos diferentes demonstrou que em todos os métodos estudados o número de respostas corretas é o mesmo ${ }^{15}$. Nesse estudo os autores sugerem a consideração dos cinco critérios seguintes, para escolha do método de avaliação da dor: a facilidade de empregar o mé- 
Tabela III - Complementação Analgésica e Escala Numérica Verbal durante o $1^{\circ}$ e $2^{\circ}$ Dias de Pós-Operatório

\begin{tabular}{|c|c|c|c|c|c|c|c|}
\hline \multirow[t]{2}{*}{ Período (h) } & \multirow[t]{2}{*}{ ENV $(\mathrm{cm})$} & \multicolumn{2}{|c|}{ Dipirona } & \multicolumn{2}{|c|}{ Tramadol } & \multicolumn{2}{|c|}{ Meperidina } \\
\hline & & $\mathrm{n}$ & $\%$ & $\mathrm{n}$ & $\%$ & $\mathrm{n}$ & $\%$ \\
\hline \multicolumn{8}{|l|}{$1^{\circ} \mathrm{PO}$} \\
\hline 0 & 3,73 & - & & - & & - & \\
\hline 1,2 & 2,77 & - & & - & & - & \\
\hline 3 & 2,64 & 2 & 18 & - & & - & \\
\hline 5 & 1,93 & 3 & 27 & 1 & 9 & - & \\
\hline 8 & 2,68 & 1 & 9 & 3 & 27 & - & \\
\hline 12 & 3,73 & 3 & 27 & 1 & 9 & - & \\
\hline \multicolumn{8}{|l|}{$2^{\circ} \mathrm{PO}$} \\
\hline 0 & 3,77 & - & & - & & - & \\
\hline 1,2 & 2,32 & 1 & 9 & - & & - & \\
\hline 3 & 2,14 & - & & - & & - & \\
\hline 5 & 2,73 & 2 & 18 & 1 & 9 & - & \\
\hline 8 & 2,23 & 3 & 27 & 2 & 18 & - & \\
\hline 12 & 3,27 & 1 & 9 & 3 & 27 & 1 & 9 \\
\hline
\end{tabular}

(n): número de pacientes; ENV: valores médios da intensidade da dor mensurada pela escala numérica verbal; $1{ }^{\circ} \mathrm{PO}$ : primeiro dia de pós-operatório; $2^{\circ} \mathrm{PO}$ : segundo dia de pós-operatório

todo, a freqüência relativa de respostas incorretas, a sensibilidade definida pelo número de categorias de respostas, a sensibilidade definida pela força estatística e a magnitude de interação entre cada escala e uma combinação linear dos índices de intensidade da dor ${ }^{15}$.

Outros autores ${ }^{16}$ compararam três escalas de dor: analógica visual, numérica e adjetiva. Observam que os pacientes preferem a escala adjetiva, porém registram que a intensidade da dor independe da preferência pelo tipo de escala.

Devido à dificuldade na mensuração da intensidade da dor, Huskisson (1974) ${ }^{17}$ sugeriu a utilização da escala analógica visual por ser um método extremamente simples, sensível e reprodutível.

Neste estudo foi empregada a escala numérica verbal, considerada eficiente para avaliação da dor pós-operatória.

A escala numérica verbal apresentou valores menores que 4,5 em todos os pacientes investigados neste estudo, mostrando que o método de analgesia utilizado foi eficaz.

Observa-se que a dor tende a diminuir com o passar do tempo, mas que, a partir de determinado momento, volta a se tornar intensa. Esse fato, possivelmente, é devido ao efeito residual do fentanil. No segundo dia a intensidade da dor foi menor que a do primeiro dia, mas sem diferença estatística $(p<0,05)$. Espera-se que a intensidade da dor tenha uma relação inversa com o tempo após a operação. O efeito residual do fentanil e o uso profilático de diclofenaco podem ter modificado os valores relacionados à intensidade da dor, apesar de a concentração plasmática residual do fentanil não se correlacionar com a intensidade de dor.

Apesar da concentração plasmática residual de fentanil estar dentro da faixa terapêutica no primeiro dia de pós-operatório ${ }^{18}$, não se observou correlação com a analgesia, embora os pacientes apresentassem dor leve. Tal fato pode ser de- vido à variação individual na intensidade da dor bem como ao uso de antiinflamatório.

Apesar de não ter sido observada correlação entre a intensidade da dor e a concentração plasmática do fentanil, sem dúvida, o conhecimento da concentração plasmática residual desse opióide é importante para a administração deste opióide assim como na escolha da medicação analgésica complementar com o prognóstico de evitar complicações.

\section{Residual Analgesic Effect of Fentanyl in Patients Undergoing Myocardial Revascularization with Cardiopulmonary Bypass}

Adriana Machado Issy, M.D., Eloísa Bonetti Espada, TSA, M.D., Rioko Kimiko Sakata, TSA, M.D., Vera Lúcia Lanchote, M.D., José Otávio Costa Auler Júnior, TSA, M.D., Silvia Regina C. J. Santos, M.D.

\section{INTRODUCTION}

Fentanyl is a potent opioid, frequently used in general anesthesia ${ }^{1,2}$. Advantages are fast effect onset with cardiovascular stability, in addition to no histamine release ${ }^{3,4}$.

Commercially available as a citrate salt in aqueous solution, fentanyl is highly liposoluble with an octanol/water partition coefficient of 816 , which is a determining factor for the entrance and exit velocity of the drug in tissue and organs, especially in the central nervous system. This penetration is 156 times faster than that of morphine ${ }^{1,5-7}$. 
High doses of fentanyl (50 to $100 \mu \mathrm{g} . \mathrm{kg}^{-1}$ ) for major surgeries deserve further investigations with regard to its residual plasma concentration contribution for postoperative analgesia.

This study aimed at evaluating the residual analgesic effect of fentanyl in the postoperative period of myocardial revascularization, as well as quantifying its plasma concentration during the same period.

\section{METHODS}

After the Ethical Committee approval and patients written consent, participated in this study 11 adult patients of both genders, with coronary failure and scheduled for myocardial revascularization. Exclusion criteria were renal, liver, endocrine, pulmonary or neuromuscular diseases. Patients should have compensated cardiac function and normal preoperative chest X-rays.

One hour before surgery, patients were premedicated with oral midazolam ( 7.5 to $15 \mathrm{mg}$ ).

Monitoring consisted of cardioscopy, pulse oximetry, invasive blood pressure, central venous pressure and bladder catheterization. Anesthesia was induced with $50 \mu \mathrm{g} . \mathrm{kg}^{-1} \mathrm{in}-$ travenous fentanyl, followed by the neuromuscular blocker (0.08 mg. kg ${ }^{-1}$ pancuronium), and was maintained with isoflurane $/ \mathrm{O}_{2}$.

Cardiopulmonary bypass was performed with total dilution with lactated Ringer's and moderate hypothermia or normothermia. Protamine was used for reversing anticoagulation until normalization of activated coagulation time (ACT).

Vasoactive drugs were used during surgery, depending on patients' hemodynamic state.

Muscular diclofenac was administered every 12 hours and the first dose was given after extubation, according to our routine.

The moment zero of the first postoperative day to evaluate analgesia and fentanyl plasma concentration was extubation. In the second postoperative day, the moment zero was set at 24 hours after moment zero of the first day. Pain was evaluated during vigorous cough and respiratory physical therapy in moments zero, $70 \mathrm{~min}, 3,5,8$ as $12 \mathrm{~h}$ of the first day, and moment zero ( $24 \mathrm{~h}$ after extubation), $70 \mathrm{~min}, 3$, 5,8 and 12 hours of the second day. Blood samples were collected at the same moments.

At every pain evaluation, patients were asked about the need for analgesic complementation. If the answer was yes, 1000 $\mathrm{mg}$ of intravenous dipyrone were administered. If pain still persisted, complementation was achieved with $100 \mathrm{mg}$ intravenous tramadol diluted in $50 \mathrm{ml}$ of $5 \%$ glucose. Patients with nausea after tramadol received intravenous methoclopramide. If pain still persisted, $30 \mathrm{mg}$ intravenous meperidine were administered.

Pain was evaluated by the Numerical Verbal Scale (NVS), which records pain intensity by asking questions during evaluation, no pain being zero and 10 the worst possible pain.
Residual fentanyl plasma concentrations were determined by radioimmunoassay in a specific $\beta$ radiation counter. Method quantification limit was $50 \mathrm{pg} \cdot \mathrm{ml}^{-1}$.

Data were submitted to statistical analysis using the GraphPad Instat and GMC software. Chi-square test was used for frequency distribution and Wilcoxon non-parametric test was used to compare pre and postoperative means and medians of variables with abnormal distribution. Significance level was $p<0.05^{8}$.

\section{RESULTS}

Participated in this study 7 males and 4 females with the mean age of $52.6 \pm 8.4$ years. Mean residual fentanyl plasma concentrations varied from 1.7 to $1.1 \mathrm{ng} \cdot \mathrm{ml}^{-1}$ in the first day and from 0.7 to $0.3 \mathrm{ng} \cdot \mathrm{ml}^{-1}$ in the second postoperative day, with statistical significance (Wilcoxon's test, $p<0.05$ ) in all moments investigated, as shown in table I.

Table I - Fentanyl Plasma Concentration (ng. $\mathrm{ml}^{-1}$ ) in the Postoperative Period

\begin{tabular}{|c|c|c|}
\hline \multirow[t]{2}{*}{ Time (h) } & \multicolumn{2}{|c|}{ Median Mean (IC95\%) } \\
\hline & $1^{\text {st }} \mathrm{PO}$ & $2^{\text {nd }} P O$ \\
\hline 0 & $\begin{array}{c}1.7 \\
1.6(1.2-2.0)\end{array}$ & $\begin{array}{c}0.5^{*} \\
0.6(0.4-0.9)\end{array}$ \\
\hline 1.2 & $\begin{array}{c}1.6 \\
1.5(1.0-1.9)\end{array}$ & $\begin{array}{c}0.7^{*} \\
0.6(0.4-0.8)\end{array}$ \\
\hline 3 & $1.4(0.6-2.6)$ & $\begin{array}{c}0.6^{*} \\
0.6(0.4-0.9)\end{array}$ \\
\hline 5 & $1.2(0.9-1.5)$ & $\begin{array}{c}0.5^{*} \\
0.4(0.3-0.7)\end{array}$ \\
\hline 8 & $1.2(0.8-1.5)$ & $\begin{array}{c}0.3^{*} \\
0.3(0.1-0.5)\end{array}$ \\
\hline 12 & $\begin{array}{c}0.8 \\
1.1(0.6-1.5)\end{array}$ & $\begin{array}{c}0.3^{*} \\
0.2\left(0.1^{-0.4)}\right)\end{array}$ \\
\hline
\end{tabular}

$1^{\text {st }}$ PO: first postoperative day, $2^{\text {nd }} \mathrm{PO}$ : second postoperative day, IC $95 \%$ : $95 \%$ confidence interval; * statistical significance, Wilcoxon's test, $p<0.05$

Pain (median) measured by the verbal visual scale varied from 1.9 to 3 in the first day and from 3.7 to 2.1 in the second postoperative day, without statistical significance (Wilcoxon's test, $p<0.05$ ) as shown in figure 1 and table II.

Table II - Pain Intensity Measured by Verbal Numeric Scale in the $1^{\text {st }}$ and $2^{\text {nd }}$ Postoperative Days

\begin{tabular}{lcc}
\hline Time $(\mathrm{h})$ & \multicolumn{2}{c}{ Median - Mean $(\mathrm{IC} 95 \%)$} \\
\hline 0 & \multicolumn{1}{c}{$1^{\text {st }} \mathrm{PO}$} & $2^{\text {nd }} \mathrm{PO}$ \\
\hline \multirow{2}{*}{1.2} & 4.0 & 3.0 \\
& $3.7(2.3-5.0)$ & $3.8(2.3-5.2)$ \\
3 & 2.0 & 2.0 \\
& $2.8(2.0-3.5)$ & $2.3(1.1-3.5)$ \\
5 & 3.0 & 2.0 \\
& $2.6(1.5-3.7)$ & $2.1(0.95-3.3)$ \\
8 & 1.0 & 2.0 \\
& $1.9(0.7-3.0)$ & $2.7(1.6-3.8)$ \\
& $2.6(1.6-3.7)$ & $2.2(0.9-3.5)$ \\
\hline
\end{tabular}

IC95\%: 95\% confidence interval, without statistical significance, Wilcoxon's test $\mathrm{p}<0.05$

Revista Brasileira de Anestesiologia Vol. 52, N 5, Setembro - Outubro, 2002 


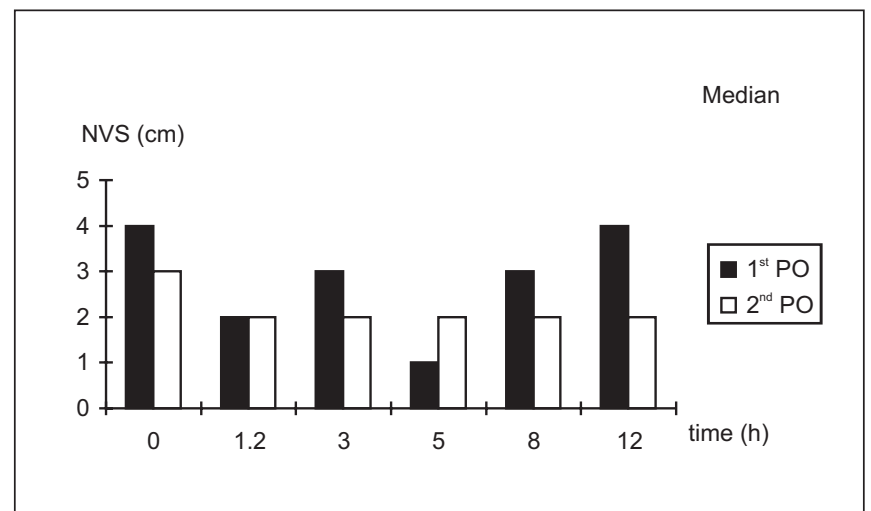

Figure 1 - Postoperative Pain Intensity ( $1^{\text {st }}$ and $2^{\text {nd }}$ days $)$ of Patients Submitted to Cardiac Surgery; without Statistical Difference

Pain intensity in the postoperative period was compared to residual fentanyl plasma concentration by the Effect versus Concentration curve, with correlation coefficients of 0.04 for the first day and of 0.0002 for the second postoperative day, as shown in figure 2 .

Table III shows the number of patients receiving complementary analgesia, type of medication, administration time and pain intensity during the first and second postoperative days.

\section{DISCUSSION}

The pre-requisite to investigate residual fentanyl plasma concentration is the availability of analytical methods with sensitivity and specificity to quantify low plasma concentrations.

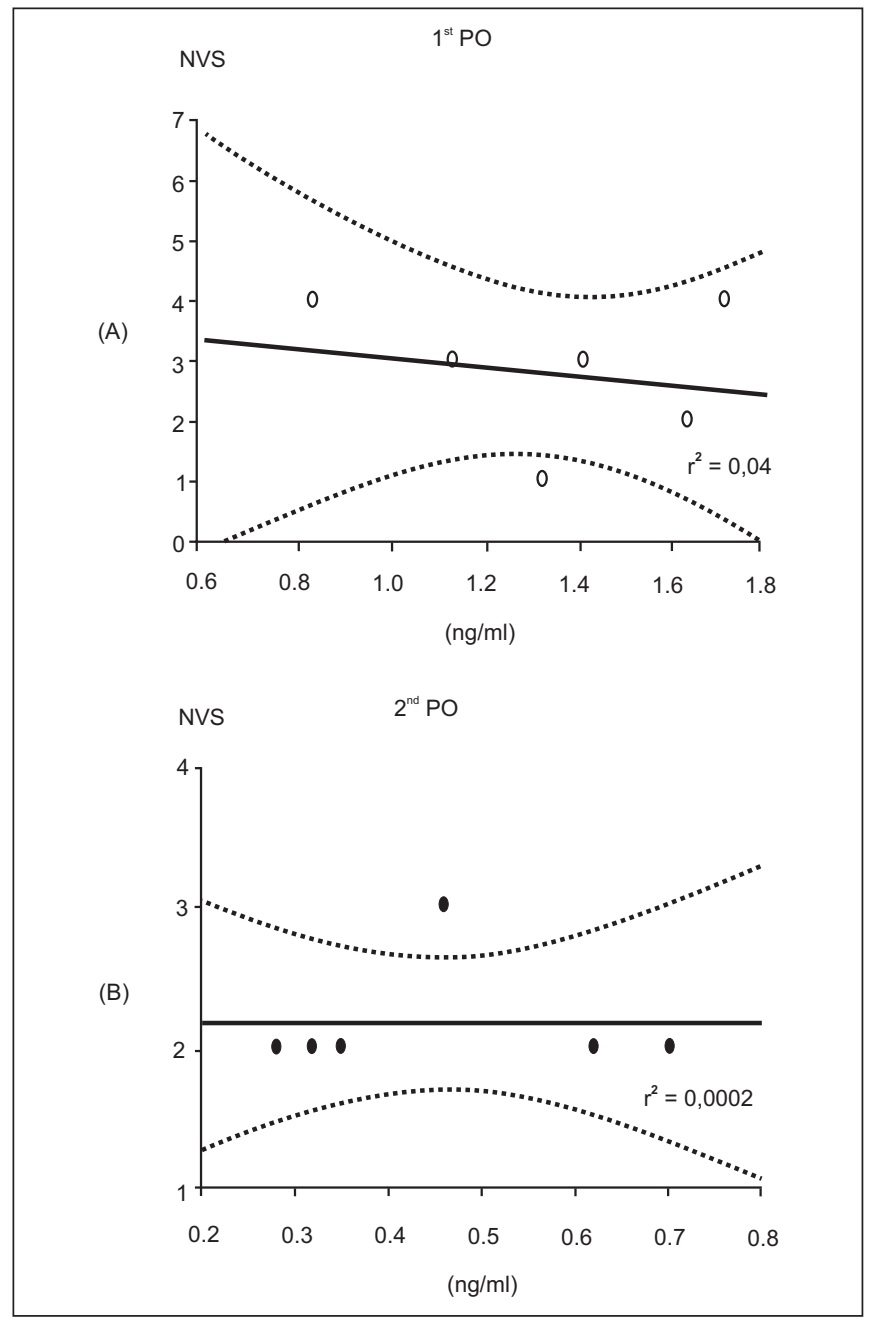

Figure 2 - Pain Intensity versus Residual Fentanyl Plasma Concentration in the First (A) and Second (B) Postoperative Days

Table III - Analgesic Supplementation and Numeric Verbal Scale During $1^{\text {st }}$ and $2^{\text {nd }}$ Postoperative Days

\begin{tabular}{|c|c|c|c|c|c|c|c|}
\hline \multirow[t]{2}{*}{ Period $(\mathrm{h})$} & \multirow[t]{2}{*}{$\mathrm{NVS}(\mathrm{cm})$} & \multicolumn{2}{|c|}{ Dipyrone } & \multicolumn{2}{|c|}{ Tramadol } & \multicolumn{2}{|c|}{ Meperidine } \\
\hline & & $\mathrm{n}$ & $\%$ & $\mathrm{n}$ & $\%$ & $\mathrm{n}$ & $\%$ \\
\hline \multicolumn{8}{|l|}{$1^{\text {st }} \mathrm{PO}$} \\
\hline 0 & 3.73 & - & & - & & - & \\
\hline 1.2 & 2.77 & - & & - & & - & \\
\hline 3 & 2.64 & 2 & 18 & - & & - & \\
\hline 5 & 1.93 & 3 & 27 & 1 & 9 & - & \\
\hline 8 & 2.68 & 1 & 9 & 3 & 27 & - & \\
\hline 12 & 3.73 & 3 & 27 & 1 & 9 & - & \\
\hline \multicolumn{8}{|l|}{$2^{\text {nd }} P O$} \\
\hline 0 & 3.77 & - & & - & & - & \\
\hline 1.2 & 2.32 & 1 & 9 & - & & - & \\
\hline 3 & 2.14 & - & & - & & - & \\
\hline 5 & 2.73 & 2 & 18 & 1 & 9 & - & \\
\hline 8 & 2.23 & 3 & 27 & 2 & 18 & - & \\
\hline 12 & 3.27 & 1 & 9 & 3 & 27 & 1 & 9 \\
\hline
\end{tabular}

(n): number of patients; NVS: mean pain intensity values measured by numeric verbal scale; $1^{\text {st }}$ PO: first postoperative day; $2^{\text {nd }}$ PO: second postoperative day 
Among the different methods mentioned in the literature, gas chromatography and mass spectrometry (GC-MS), although having adequate sensitivity, specificity and linearity, has some disadvantages as compared to radioimmunoassay (RIE), such as: high cost, labor-intensive sample preparation and the need for twice the biological material in the collection process $^{49}$.

Currently, radioimmunoassay has been considered the method of choice for opioid dosage not only for its high sensitivity, but also for its easy and fast execution, with less analytical errors. In this technique, choice, execution and standardization of procedures are critical for precise and accurate results ${ }^{4,10}$

In our study, all patients were submitted to myocardial revascularization with cardiopulmonary bypass, which in general involves the placement of 2 to 3 chest drains ${ }^{11,12}$. According to clinical experience based on patients' complaints, these drains are responsible for severe pain.

Although subjective, pain may be evaluated by scales which quantify its intensity. Some authors ${ }^{13,14}$, also highlight the care to be taken in choosing the method for pain evaluation.

A comparative study with six different methods has shown that the number of correct answers was the same ${ }^{15}$, and the authors suggest the following criteria for choosing the pain evaluation method: easiness of the method, relative frequency of wrong answers, sensitivity defined by the number of categories of answers, sensitivity defined by statistical strength and the magnitude of interaction between each scale; and a linear combination of pain intensity ratios ${ }^{15}$. Other authors ${ }^{16}$ have compared three pain scales: visual analog, numeric and adjective and have observed that patients prefer the adjective scale, although pain intensity being independent of the type of scale.

Due to the difficulty in evaluating pain intensity, Huskisson $(1974){ }^{17}$ has suggested the visual analog scale for being an extremely simple, sensitive and reproducible method.

Our study used the numeric verbal scale, considered effective in evaluating postoperative pain

The numeric verbal scale has shown values below 4.5 in all patients, showing that the analgesic method used was effective.

Pain tends to decrease with time, but as from a certain moment it becomes again severe. This is likely due to the residual effect of fentanyl. Pain was less severe in the second day as compared to the first day, but without statistical difference $(p<0.05)$. A reverse relationship between pain and time after surgery is to be expected. Residual fentanyl effect and the prophylactic use of diclofenac may have changed pain intensity, although residual fentanyl plasma concentration was not correlated to pain intensity.

Residual fentanyl plasma concentration was within the therapeutic range in the first postoperative day ${ }^{18}$, but there has been no correlation with analgesia, although patients referred only mild pain. This may be due to an individual variation in pain intensity, as well as to the anti-inflammatory drug.
Although no correlation was observed between pain intensity and fentanyl plasma concentration, clearly the knowledge of its residual plasma concentration is important for its administration, as well as for the choice of supplementary analgesia with the aim of preventing complications.

\section{REFERÊNCIAS - REFERENCES}

01. Bovill JG - Pharmacokinetics of Opioids, em: Bowdle TA, Horita A, Kharasch? The Pharmacologic Basis of Anesthesiology. New York: Churchill Livingstone, 1994;37-81.

02. Bovill JG, Boer F - Opioids in Cardiac Anesthesia, em: Kaplan JA - Cardiac Anesthesia. $3^{\text {rd }}$ Ed, Philadelphia, WB. Saunders, 1993;467-509.

03. Flacke JW, Flacke WE, Bloor BC - Histamine release by four narcotics: a double-blind study in humans. Anesth Analg, 1987;66:723-730.

04. Fryirsa B, Woodhouse A, Huang JL et al. - Determination of subnanogram concentrations of fentanyl in plasma by gas chromatography-mass spectrometry: comparison with standard radioimmunoassay. J Chromatogr Biomed Appl, 1997;688: 79-85.

05. Bower S, Hull CJ - Comparative pharmacokinetics of fentanyl and alfentanil. Br J Anaesth, 1982;54:871-877.

06. Resine T, Pasternak G - Opioid Analgesics and Antagonists, em: Goodman \& Gilman's - The Pharmacological Basis of Therapeutics. $9^{\text {th }}$ Ed, New York; McGraw-Hill, 1996;521 -555.

07. Scholz J, Steinfath M, Schulz M - Clinical Pharmacokinetics of alfentanil, fentanyl and sufentanil: an update. Clin Pharmacokinet, 1996;31:275-292.

08. Sachs L - Applied Statistics: A Handbook of Techniques. $2^{\text {nd }}$ Ed, Springer-Verlag, 1984;111-134.

09. Vanrooy HH, Vermeulen NPE, Bovill JG -The assay of fentanyl and its metabolites in plasma of patients using gas chromatography-mass spectrometry. J Chromatography, 1981;223:86-93.

10. Scott JC, Stanski DR - Decreased fentanyl and alfentanil dose requirements with age: a simultaneous pharmacokinetic and pharmacodynamic evaluation. J Pharmacol Exp Ther, 1987; 240:159-163.

11. Sebel PS, Bovill JG, Boekhorst RA et al - Cardiovascular effects of high-dose fentanyl anesthesia. Acta Anaesthesiol Scand, 1982;26:308-315.

12. Bovill JG, Sebel PS - Pharmacokinetics of high-dose fentanyl. Br J Anaesth, 1980;52:795-801.

13. Gracely RH, Dubner R - Pain assessment in humans - a reply to hall. Pain, 1981;11:109-129.

14. Paige D, Cioffi AM - Pain Assessment and Measurement, em: Sinatra RS, Hord AH, Ginsberg B et al - Acute Pain Mechanisms and Management. Saint Louis, Mosby, 1992;70-77.

15. Jensen MP, Karoly P, Braver S - The measurement of clinical pain intensity: a comparison of six methods. Pain, 1986;27: 177-226.

16. Kremer E, Atkinson JA, Ignelzi RJ - Measurement of pain: patient preference does not confound pain measurement. Pain, 1981;10:241-248.

17. Huskisson EC - Measurement of pain. Lancet, 1974;2: 1127-1131.

18. Benet LZ, Qie S, Schwartz JB - Design and Optimization of Dosage Regimens Pharmacokinetic Data, em: Goodman \& Gilman's - The Pharmacological Basis of Therapeutics. $9^{\text {th }}$ Ed, New York: McGraw-Hill, 1996;1707-1792. 


\section{RESUMEN}

Issy AM, Espada EB, Sakata RK, Lanchote VL, Auler Jr JOC, Santos SRCJ - Efecto Analgésico Residual del Fentanil en Pacientes Sometidos a Revascularización del Miocárdio con Circulación Extracorpórea

Justificativa y Objetivos - A pesar del uso frecuente del fentanil en altas dosis para operaciones de grande porte, la contribución de la concentración plasmática residual de ese fármaco sobre la analgesia pós-operatoria merece mayores investigaciones. El objetivo de este estudio es evaluar el efecto analgésico del fentanil residual en el primero y segundo día después de revascularización miocárdica, bien como dosificar su concentración.

Método - Fueron investigados 11 pacientes sometidos a revascularización del miocárdio con circulación extracorpórea, bajo anestesia general empleando $50 \mu \mathrm{g} . \mathrm{kg}^{-1}$ de fentanil, por vía venosa. Se evaluó la analgesia por la escala numérica ver- bal en los tiempos cero (extubación), 70 minutos, 3, 5, 8 y 12 horas en el primero día y en los tiempos cero (24 horas después de extubación), 70 minutos, 3, 5, 8 y 12 horas en el segundo día. El dolor fue evaluado mediante tos vigorosa y fisioterapia respiratoria. A cada mensuración del dolor, se cuestionó sobre la necesidad del paciente recibir complemento analgésico. Las muestras plasmáticas del fentanil fueron colectadas en los tiempos cero, 70 minutos, 3, 5, 8 y 12 horas del primero y segundo día de pós-operatorio y mensuradas por el método de radioinmunoensayo.

Resultados - La intensidad del dolor varió en media de 1,9 a 3,7 en el primero día y de 2,1 a 3,8 en el segundo día de pós-operatorio. Los niveles plasmáticos de fentanil (> $1 \mathrm{ng} / \mathrm{ml}$ ), evidenciaron su contribución en la analgesia en el primero día después de la operación.

Conclusiones - A pesar de no haber sido observada correlación entre la concentración plasmática residual de fentanil y la intensidad del dolor, los pacientes presentaron dolor leve durante todo el período investigado $(E N V<4,5)$. 